\title{
MOLECULAR-DYNAMIC MODELING OF THERMOPHYSICAL PROPERTIES OF PHONON SUBSYSTEM OF COPPER IN WIDE TEMPERATURE RANGE
}

\author{
M.M. DEMIN, O.N. KOROLEVA*, A.A. ALEKSASHKINA, V.I. MAZHUKIN \\ Keldysh Institute of Applied Mathematics, Russian Academy of Science. \\ *Corresponding author. E-mail: koroleva.on@mail.ru
}

DOI: $10.20948 /$ mathmontis-2020-47-12

\begin{abstract}
Summary. Copper is a noble metal and has unique properties, due to which it is widely used in scientific research, industrial production and, more recently, in the problems of biomedicine. Using the molecular dynamics method, a series of calculations was performed to determine the lattice thermophysical properties of copper in a wide temperature range of $300 \mathrm{~K} \leq \mathrm{T} \leq 5800 \mathrm{~K}$. In the calculations, special attention is paid to the melting-crystallization and near-critical regions, in which cardinal changes in the thermophysical properties of the substance occur. The temperature dependences of the specific heat $C p(T)$, thermal conductivity $\kappa(T)$, and density $\rho(T)$ were among the studied characteristics of the phonon subsystem of $\mathrm{Cu}$. Molecular - dynamic modeling was carried out using the potential of the embedded atom method (EAM). A comparison of the results with the results of experiments and alternative calculations showed a good agreement. The obtained calculation results were approximated by polynomials of low degrees..
\end{abstract}

\section{INTRODUCTION}

Copper, a noble metal, occupies an important place among metals in importance and prevalence in many branches of scientific research and innovative technological applications [1-5]. Such a relatively new, rapidly developing direction is the production of metal-based nanomaterials, including copper, which attracts general attention by its wide applicability [58]. Due to its unique properties, copper nanoparticles have gained the possibility of being used primarily in problems of theranostics and nanomedicine [1-3, 5]. One of the rapidly developing methods for the production of nanomaterials is pulsed laser ablation of metals (PLA) [5-9]. The increasing possibilities of using PLA in the production of nanoparticles make this direction attractive for basic research, the main tool of which is mathematical modeling. Continuous, atomistic, and combined models are used for the mathematical description of fast and highly nonequilibrium processes induced by ultrashort laser pulses in the metal targets [9-15]. For continuum and combined models, the properties of the substance are input parameters. Therefore, one of the most important problems of mathematical modeling is the need to determine for each of the subsystems the thermophysical, optical, and thermodynamic characteristics in a wide temperature range - from room temperature $T_{0}=300$ $\mathrm{K}$ to critical $\mathrm{T}_{\text {cr. }}$.

The most important thermophysical properties characterizing heat transfer in metals are density $\rho(T)$, specific heat $C p(T)$ and thermal conductivity $\kappa(T)$. 
The specific heat of metals at the temperature $\mathrm{T}>300 \mathrm{~K}$ is mainly determined by the lattice vibrations. The contribution of the electronic subsystem to the specific heat is noticeable only at a low temperature $\mathrm{T}<10 \mathrm{~K}[16]$.

Unlike specific heat, the contribution of the electronic component to the thermal conductivity of copper is significant. According to [17], the contribution of the electronic component to the total thermal conductivity is $\approx 95 \%$. Since in metals the main part of the heat flux is carried by conduction electrons, it was believed that lattice thermal conductivity does not play a significant role. Therefore, it was not necessary to separate the total thermal conductivity into electronic and phonon components.

The interest in quantifying the thermophysical characteristics of the phonon subsystem of metals was stimulated primarily by the need for a deeper understanding of the mechanisms of thermal transfer during nonequilibrium energy transfer in a number of applications, for example, $[18,19]$.

Due to the limited possibilities of instrumental measurement of the thermophysical characteristics of the material under study at high temperatures $\left(T>T_{m}\right.$, where $T_{m}$ is the melting temperature), computational approaches become relevant.

Significant progress, first of all, in the development of (numerical) atomic modeling methods (and computational algorithms) allows one to determine the thermophysical characteristics (phonon specific heat, phonon thermal conductivity, etc.) in a wide temperature range for most metals [20-30] and semiconductors [24, 25, 31-34] with a sufficient degree of accuracy.

The aim of this work is to obtain lattice thermophysical characteristics $(\rho(T), C p(T), \kappa(T))$ of copper in a wide temperature range $T_{0} \leq T \leq T_{c r}$ using the molecular dynamics method with the EAM potential [21].

\section{METHODS AND APPROACHES}

The determination of the thermophysical properties of the phonon subsystem of copper in this work is based on the atomistic approach. Atomistic models rely on the molecular dynamics (MD) method. The MD method is based on a model representation of a polyatomic molecular system in which all atoms are represented by material points, and the motion is described in the classical case by Newton's equations. Because of this, atomistic models are a system of differential equations, the integration of which requires knowledge of the coordinates and velocities of all particles at the initial time $t=0$. The resulting ODE system is solved using the Verlet finite-difference scheme [35].

When using atomistic models to study various properties of substances, the most important role is played by the choice of interaction potentials between particles, since the reliability of the results obtained directly depends on it. In molecular dynamics modeling of the properties of metals, the empirical and semi-empirical potentials of the "embedded atom method" (EAM) are mainly used as the interparticle interaction potentials [22, 23]. Since pair and collective interactions are taken into account in the EAM potentials, the potential energy of the metal is the sum of the embedding potential of the ith atom, which depends on the effective electron density in the region where the center of the atom and the pair potential are located. However, the EAM potentials do not take into account the phonon-electron interaction, which is their drawback. The disadvantages of these potentials include a large number of fitting parameters included in them (up to two dozen). When choosing the potential 
for atomistic modeling, careful testing of the potential used is necessary, since not all the EAM potentials used allow a good description of both the crystalline and liquid phases of the metal. In this work, we used the EAM potential for copper developed and tested in [21].

Of all the thermophysical properties, the determination of phonon thermal conductivity in the framework of classical molecular dynamics occupies a special place, being a complex problem.

The direct method (DM) was chosen as an approach for determining the temperature dependence of the phonon thermal conductivity of copper using molecular dynamics (MD). This method is the most simple and economical from a computational point of view.

The direct method (DM) [24-26] is one of the most common methods for calculating thermal conductivity. DM is a nonequilibrium method of molecular dynamics (NEMD); it is based on applying a temperature gradient to the modeling cell, for which it received its name "heat source - sink". Due to this, the direct method is similar to the experimental situation. One of the advantages of DM is the saving of computing resources, which is very important, sometimes determining the choosing of the modeling method. For example, as noted in [24], for the direct method, the simulation time of $1 \mathrm{~ns}$ is sufficient to obtain a smooth temperature profile, and the value of $\kappa$ converges with an accuracy of $\pm 10 \%$. The method demonstrates the finite-size effects, which are its drawback. These effects arise if the mean free path of phonons is comparable to the size of the simulation cell. In this regard, the necessary size of the computational domain to achieve a completely convergent value of $\kappa$ may be beyond the reach of atomistic modeling and it becomes necessary to impose a restriction on the smallest length of the computational domain. In this connection, the thermal conductivity of copper can be obtained by the direct method from modeling systems of different sizes and extrapolating the results to a system of an infinite size.

Along with classical molecular dynamics, the ab-initio approach is also used to determine the phonon thermal conductivity $[17,36]$. The ab initio methods have appeared recently and are considered the most promising. They do not require specifying the interparticle potential and can be applied to any material. However, ab-initio methods have limitations associated with an increase in computational costs with an increase in model size. The use of these methods in the calculation of phonon thermal conductivity allows one to take into account the influence of both phonon-phonon (p-p) and phonon-electron (p-e) interactions, which can significantly increase the reliability of the results. However, the number of calculations of the phonon thermal conductivity of metals and, in particular, copper [17, 36], is currently relatively small. As a rule, all calculations are limited to the solid-state phase in the temperature range $T \sim(300-1000) \mathrm{K}$. There are no systematic results of experimentaltheoretical studies of the properties of liquid metals in a wide temperature range (from the beginning of melting to the critical region).

The wide temperature range $300 \mathrm{~K} \leq T \leq 5800 \mathrm{~K}$, in which the thermophysical properties of copper are determined in this work, covers the first-order phase transition (meltingcrystallization) and the near-critical region in which drastic changes in the thermophysical properties of the substance occur. Therefore, calculations of the properties of copper in this range cannot be carried out without knowledge of such important characteristics as the melting temperature $T_{m}$ and critical parameters: temperature $T_{c r}$, density $\rho_{c r}$, pressure $P_{c r}$.

The equilibrium melting temperature used in the calculations was obtained from molecular dynamics calculations in [27] with the EAM potential [21], which is also used in this work, by the two-phase method [28]. We used a system with an ensemble of particles of 8000 atoms. 
The obtained value is $T_{m}=1330 \mathrm{~K}$, slightly lower than the reference $\left(T_{m}=1356 \mathrm{~K}\right)$ [37] and experimental value $\left(T_{m}=1357.7 \mathrm{~K}\right)[38]$ and deviates from these values by $1.9 \%$ and $2.06 \%$, respectively. The error is quite acceptable for modeling. In this work, the following values of the critical parameters of copper were taken: $T_{c r} \approx 6550 \mathrm{~K}, \rho_{c r} \approx 1.895 \mathrm{~g} / \mathrm{cm} 3, P_{c r} \approx 0.16$ $\mathrm{GPa}$, obtained in [30] using the liquid - vapor coexistence curve.

The specific heat and thermal conductivity of the phonon subsystem, as well as the copper density characterizing heat transfer, were simulated in the temperature range $300 \leq \mathrm{T} \leq 5800$ $\mathrm{K}$ using the well-known LAMMPS application package (large-scale atomic-molecular massively parallel simulator) [39]. It implements many paired and multiparticle potentials, the ability to save atomic configurations in a text file, as well as built-in thermostats and barostats. The velocity and pressure for the ensemble of particles were controlled using the thermostat and barostat of Berendsen [40].

\section{MODELING RESULTS}

For convenience of further use, the calculation results are approximated by polynomials of the degree of $\mathrm{m}$ :

$$
P_{\mathrm{m}}(\mathrm{x})=\sum_{k=0}^{m} a_{k} x^{k}
$$

where $a_{k}$ are the polynomial coefficients.

The approximation error was calculated by the least squares criterion [41]:

$$
\Delta\left(P_{m}\left(t_{j}\right), y_{j}\right)=\sqrt{(1 / n+1) \sum_{j=0}^{n}\left(P_{m}\left(t_{j}\right)-y_{j}\right)^{2}} \rightarrow \text { min },
$$

where $y_{j}$ are the values of the variable from the results of calculations for $t_{j}(j=0, \ldots, n)$.

\subsection{Calculation of specific heat and density of copper}

The traditional way to determine the thermophysical properties of metals is experiment. For copper, the experimental values of density [42-44] and heat capacity in the solid $[45,46]$ and liquid [46, 47] phases are known. The experimental approach has limitations, primarily for the temperature range. The copper density was obtained experimentally [42] in a wide temperature range of $300 \mathrm{~K} \leq T \leq 5000 \mathrm{~K}$, and the heat capacity of the liquid phase was obtained up to $2000 \mathrm{~K}$ [46]. A scatter of values is also observed in various experiments, which was noted in [44].

However, when simulating the laser ablation processes, the modeling enters the higher temperature region, including the region of the critical point, so the known data becomes insufficient. In addition, for working with mathematical models, it is relevant to obtain temperature dependences in a wide range of parameters.

The temperature dependences of the density $\rho(T)$ and specific heat $C_{p}(T)$ of copper were determined from a series of molecular dynamics calculations within the framework of one computational experiment.

We used a cubic computational region of $30 \times 30 \times 30$ unit cells containing an fcc crystal of 108,000 particles (fluctuations that are too large arise with smaller sizes). Periodic boundary conditions were set. The relaxation procedure preceding the simulation was carried out at a 
temperature of $300 \mathrm{~K}$ and zero pressure. After that, the slow heating of the sample with a constant rate of approximately $0.5 \mathrm{~K} / \mathrm{ps}$ continued to a temperature of $6000 \mathrm{~K}$. During MD calculations, the temperature dependences were recorded: density $\rho(T)$ and enthalpy $H(T)$. The experiment was carried out at a constant zero pressure $P=0$.

\subsubsection{Density of copper}

As a result of MD calculations in the range $300 \mathrm{~K}<T<5620 \mathrm{~K}$, the temperature dependence of the density of copper $\rho(T)$ was obtained, which, after additional statistical processing, is shown in Figure 1. The markers in this figure show the experimental data [42]. The vertical dashed lines indicate the melting temperature $T_{m}$ [27] and the critical temperature $T_{c r}$ [30] of copper. Figure 1 shows the changes in the density of copper at the equilibrium melting temperature $\left(T_{m}=1330 \mathrm{~K}\right)$. The density of the copper melt is lower than the density of the crystal at the same temperature $T_{m}$, i.e. copper melts with decreasing density, similar to what was observed in experiments $[42,43]$. At the equilibrium melting temperature $T_{m}$, the density changes abruptly. The density difference between the solid and liquid phases in the calculations is $5.2 \%$, and in the experiment [42] - 4.4\%, which shows a fairly good agreement between the results.

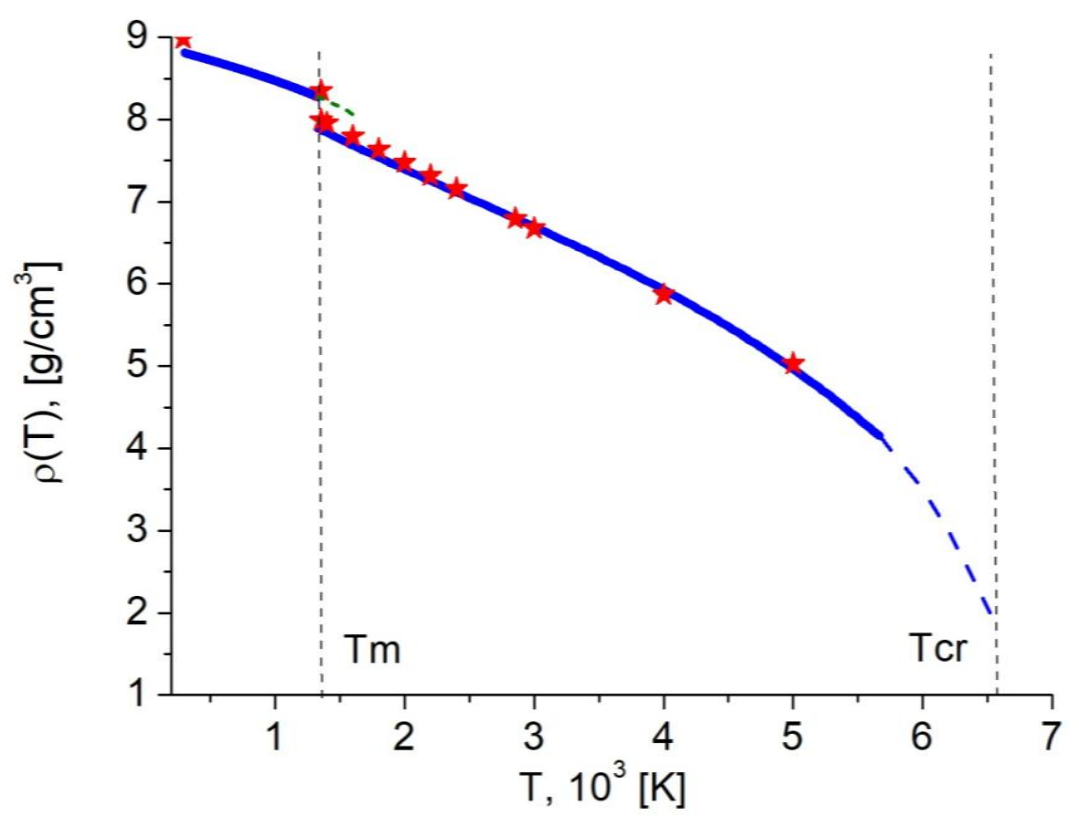

Fig. 1. The temperature dependence of the density of copper. The markers show the experimental data [42].

It is also noticeable that the density decreases to $\rho(T) \approx 8 \mathrm{~g} / \mathrm{cm}^{3}$ upon overheating of the solid phase in the temperature range $T_{m}<T<1.2 T_{m}$ (green dashed line in Figure 1). In the liquid phase, with increasing temperature $T>T_{m}$, the copper density decreases. The results obtained show good agreement with the experimental results [42]. At $T \approx 3000 \mathrm{~K}$, the density value in this work is $\rho(T)=6.678 \mathrm{~g} / \mathrm{cm}^{3}$, in the experiment $-\rho(T)=6.675 \mathrm{~g} / \mathrm{cm}^{3}$ the values differ by $0.04 \%$. At $T \approx 5000 \mathrm{~K}$ in this work, $\rho(T)=4.956 \mathrm{~g} / \mathrm{cm}^{3}$, in the experiment $-\rho(T)=$ $5.03 \mathrm{~g} / \mathrm{cm}^{3}$, the difference is $1.49 \%$. At $T \approx 5620 \mathrm{~K}$, a temperature close to the critical 
temperature $T_{c r}=6550 \mathrm{~K}[30]$, the density $\rho(T)=4.207 \mathrm{~g} / \mathrm{cm}^{3}$ was obtained. In Fig. 1, the dashed line shows the continuation of the function $\rho(T)$, which extrapolates the density of copper to the critical point. At $T_{c r}=6550 \mathrm{~K}$, the density $\rho_{c r} \approx 1.895 \mathrm{~g} / \mathrm{cm}^{3}$ was obtained in [30].

The results obtained in the calculations are more convenient to use as an analytical dependence of the form (1). For solid and liquid phases, dependences of the third degree are obtained

$$
\rho(T)=a_{0}+a_{1}\left(T-T_{0}\right)+a_{2}\left(T-T_{0}\right)^{2}+a_{3}\left(T-T_{0}\right)^{3}
$$

For the solid phase, $\left(300 \mathrm{~K} \leq T \leq T_{m}\right), T_{0}=300 \mathrm{~K}$, for the liquid phase, $\left(T_{m} \leq T \leq 5620 \mathrm{~K}\right)$, $T_{0}=T_{m}$. The values of the coefficients $a_{k}$ and the approximation errors by the least squares criterion (2) are presented in table 1.

\begin{tabular}{|l|l|l|}
\hline$k$ & Solid & Liquid \\
\hline$a_{0}$ & 8,81 & 7.89 \\
\hline$a_{1}$ & $-4.28 \times 10^{-4}$ & $-7.96 \times 10^{-4}$ \\
\hline$a_{2}$ & $-6.12 \times 10^{-8}$ & $8.69 \times 10^{-8}$ \\
\hline$a_{3}$ & $-2.77 \times 10^{-11}$ & $-2,383 \times 10^{-11}$ \\
\hline$\Delta\left(P\left(x_{j}\right), y_{j}\right)$ & 0.001 & 0.014 \\
\hline
\end{tabular}

Table 1. The coefficients $a_{k}$ of the function, which approximates the calculation results of the copper density $\rho(T)$ in $\mathrm{g} / \mathrm{cm}^{3}$.

\subsubsection{Specific heat of copper}

The temperature dependence of the specific heat of the lattice $C_{p}(T)$ in the temperature range $300 \mathrm{~K}<T<5800 \mathrm{~K}$ at constant pressure $P$ in this work was determined from the enthalpy $H(T)$ obtained during the computational MD experiment considered above. The values of $H(T)$ were approximated for the liquid and solid phases by polynomials $\tilde{H}(T)(1)$. The temperature dependence of the specific heat $C_{p}(T)$ for each phase was determined by differentiating the corresponding dependence $\tilde{H}(T)$ :

$$
C_{p}(T)=\left(\frac{\partial \tilde{H}(T)}{\partial T}\right)_{P}
$$

Fig. 2. shows the temperature dependence of the specific heat of copper $C_{p}(T)$ according to the results of calculations of the present work, the markers show the reference and experimental results $[37,38]$. The vertical dashed lines indicate the melting temperature $T_{m}$ and the critical temperature $T_{c r}$ of copper. The region of the solid - liquid phase transition, with zooming, is shown in the inset of Fig. 2. It is seen that at the equilibrium melting temperature $T_{m}$, an insignificant jump by about $\sim 3.128 \%$ occurs, an abrupt decrease in the heat capacity of copper. According to the experiment [38], this value is $\sim 1.529 \%$. On the inset of fig. 2 one can clearly see an increase in the specific heat to $\mathrm{C}_{\mathrm{p}}(\mathrm{T}) \approx 39 \mathrm{~J} \cdot \mathrm{mol}^{-1} \cdot \mathrm{K}^{-1}$ upon overheating of the solid phase in the temperature range $T_{m}<T<1.2 T_{m}$ (green dashed 
line in Figure 2). With increasing temperature $T_{m}<T<2.63 T_{m}$ in the liquid phase, the specific heat is almost constant and amounts to $C_{p}(T) \approx 31.0 \mathrm{~J} \cdot \mathrm{mol}^{-1} \cdot \mathrm{K}^{-1}$, which is $8 \%$ less than $C_{p}(T)$ $\approx 33.84$ [38]. At the temperature $T>4000 \mathrm{~K}$, the specific heat of copper increases. At $T=$ $5800 \mathrm{~K}$, its value is $C_{p}(T)=47.698 \mathrm{~J} \cdot \mathrm{mol}^{-1} \cdot \mathrm{K}^{-1}$. In the near-critical region in Fig. 2, the dashed line shows the extrapolation of the temperature dependence of the specific heat to the critical point.

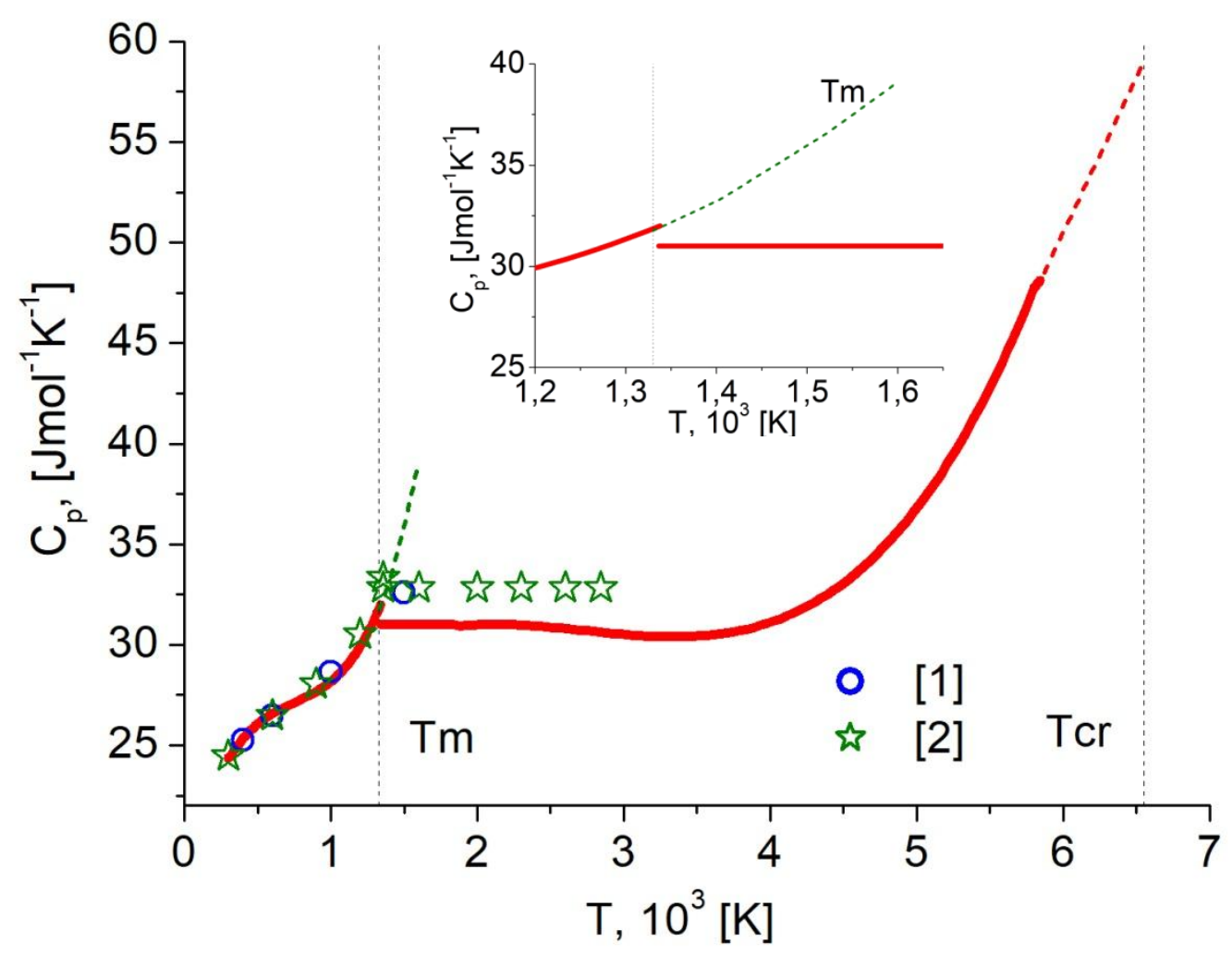

Fig.2. The temperature dependence of the specific heat $\mathrm{Cp}(\mathrm{T})$ of copper according to the calculation results (solid line). Markers: (1), (2) - experimental results [37, 38].

For the use in further calculations, the results obtained are more convenient to use in the form of an analytical dependence of the form (1). For the solid and liquid phases, the results were approximated by power dependences of the 4th degree:

$$
C_{p}(T)=a_{0}+a_{1}\left(T-T_{0}\right)+a_{2}\left(T-T_{0}\right)^{2}+a_{3}\left(T-T_{0}\right)^{3}+a_{4}\left(T-T_{0}\right)^{4},
$$

where $T_{0}=300 \mathrm{~K}$ for the solid phase $\left(300 \mathrm{~K} \leq T \leq T_{m}\right), T_{0}=T_{m}$ for the liquid phase $\left(T_{m} \leq T \leq\right.$ $5800 \mathrm{~K})$. The values of coefficients $a_{k}$ and approximation error according to the least squares criterion (2) are shown in the Table 2. 


\begin{tabular}{|l|l|l|}
\hline \multicolumn{1}{|c|}{$\mathrm{k}$} & Solid & Liquid \\
\hline$a_{0}$ & 24.27 & 31.0018 \\
\hline$a_{1}$ & $1.23 \times 10^{-2}$ & $3.28 \times 10^{-3}$ \\
\hline$a_{2}$ & $-2.05 \times 10^{-5}$ & $-2.91 \times 10^{-6}$ \\
\hline$a_{3}$ & $1,53 \times 10^{-8}$ & $7.06 \times 10^{-10}$ \\
\hline$a_{4}$ & $-2.88 \times 10^{-12}$ & $-4.68 \times 10^{-15}$ \\
\hline$\Delta\left(P\left(x_{j}\right), y_{j}\right)$ & 0.021 & 0.038 \\
\hline
\end{tabular}

Table 2. The values of coefficients $a_{k}$ for the function, which approximates the calculation results of specific heat of copper $C_{p}(T) \mathrm{J} \cdot \mathrm{mol}^{-1} \cdot \mathrm{K}^{-1}$

\subsection{Calculation of thermal conductivity of copper}

To determine the thermal conductivity of the phonon subsystem of copper, a series of calculations was carried out based on molecular dynamics modeling. The phonon thermal conductivity was determined using the direct method (DM) [24.25].

When using DM, the heat source and sink areas are created in the modeling domain to apply a constant heat flux along the direction of interest.

At each time step, a fixed amount of heat $d Q_{N}$ was deposited in the heating region, and the same amount was taken from the sink region. The heat flux $\mathrm{W}$ was calculated as

$$
W=d Q /(S N d t) / 2 \text {, }
$$

where $d Q=N \times d t \times \delta Q_{N}$ is the total deposited energy, $\delta Q_{N}$ is the energy deposited during one timestep, $N$ is the number of timesteps, $d t$ is the size of the timestep, $S$ is the domain crosssection. The timestep size was chosen depending on the temperature, from $3 \mathrm{fs}$ at $300 \mathrm{~K}$ to $1 \mathrm{fs}$ at $2000 \mathrm{~K}$ and above. The division by 2 is used due to periodic boundary conditions, i.e. heat flux goes in two directions. Then, the resulting temperature gradient is calculated, and the thermal conductivity coefficient $\kappa_{\text {lat }}$ was determined from the known heat flux by the Fourier law [16]

$$
W=-\kappa_{\text {lat }} \frac{\partial T}{\partial x}
$$

where $W$ is the heat flux, $x$ is the coordinate in the direction of the flux.

The difficulty in applying the direct method to solids lies in the fact that the size of the modeling region should be much larger than the mean free path of phonons in a substance. For a crystal, this is difficult to do, because requires a very large size of the computational domain and, accordingly, a very large number of atoms. Therefore, when calculating with a small number of atoms, the thermal conductivity coefficient is dependent on the length of the region due to phonon scattering at the boundary. To limit the size of the simulation region, a scaling procedure is used, in which the thermal conductivity is determined for several lengths of the simulation region $L_{n}$ ( $\mathrm{n}$ is the number of unit cells in the computational domain) along the $\mathrm{x}$ direction. Then, the inverse dependence of the thermal conductivity $1 / \kappa_{\text {lat }}$ is constructed with respect to the reciprocal of the length of the simulation region, $1 / L_{n}$, and the thermal conductivity is determined by extrapolating the data $1 / L_{n} \rightarrow 0$ [24-26]. 
To determine the thermal conductivity of copper, the simulation domain in the form of a parallelepiped was considered. The initial sizes of the region were $10 \times 10 \times 20$ unit cells (lattice constant $0.361 \mathrm{~nm}$ ), corresponding to 8000 particles. Periodic boundary conditions were set along the three axes. As the interaction potential, the EAM potential is used [21]. The particle velocities were set as random variables with a Maxwell distribution corresponding to a double temperature of $600 \mathrm{~K}$. Then the sample was equilibrated at $300 \mathrm{~K}$ using a thermostat and barostat.

The sample was divided along the $\mathrm{x}$ axis into the number of cells corresponding to the number of particles. At each step, a certain amount of heat is deposited in the first interval, and the same amount of heat is taken from the middle of the sample, where the drain is located. After some time (for small samples $5 \mathrm{~ns}$, for large $10 \mathrm{~ns}$ ), a stationary equilibrium is established. The temperature difference is calculated at 0.8 of the entire length between the heat source and the sink over the last $0.5 \mathrm{~ns}$ and averaged.

\subsubsection{Modeling results of thermal conductivity}

To calculate the thermal conductivity from the Fourier law (5), it is necessary to determine the value of the heat flux $W$ (4) from the spatial temperature distribution obtained from the MD modeling. Figure 3 shows the time-averaged spatial temperature profile for an average temperature of $300 \mathrm{~K}$. In a small region $(\sim 6 \mathrm{~nm})$ in the immediate vicinity of the source, a very strong nonlinear temperature profile is observed. The same strong nonlinear temperature profile is also observed near the sink in the middle of the computational domain. In the intermediate region, the behavior of the temperature profile is close to a linear dependence. The intermediate region in Fig. 3 is indicated by dashed lines. In this interval between the heat source and sink, the temperature gradient was measured. The presence of the heat source and heat sink, the use of periodic boundary conditions creates a current in two opposite directions.

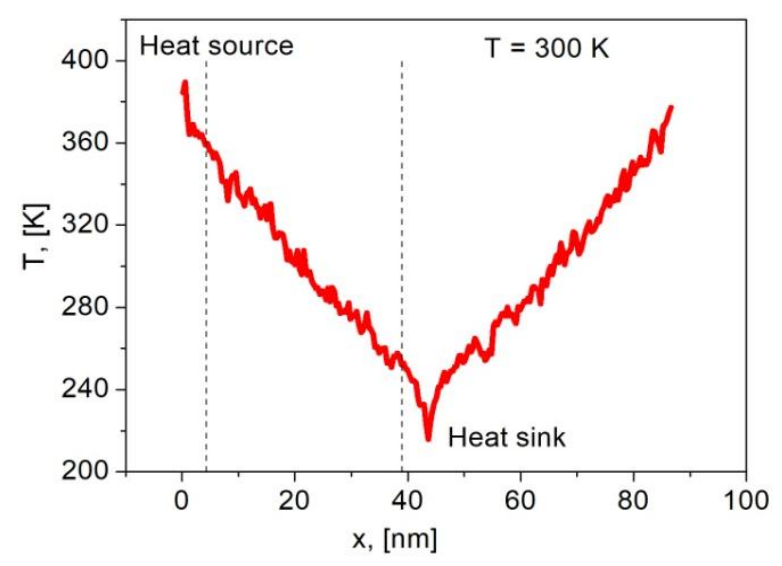

Fig. 3. Spatial temperature profile at one moment in time. The dashed lines indicate the interval in which the temperature gradient was measured.

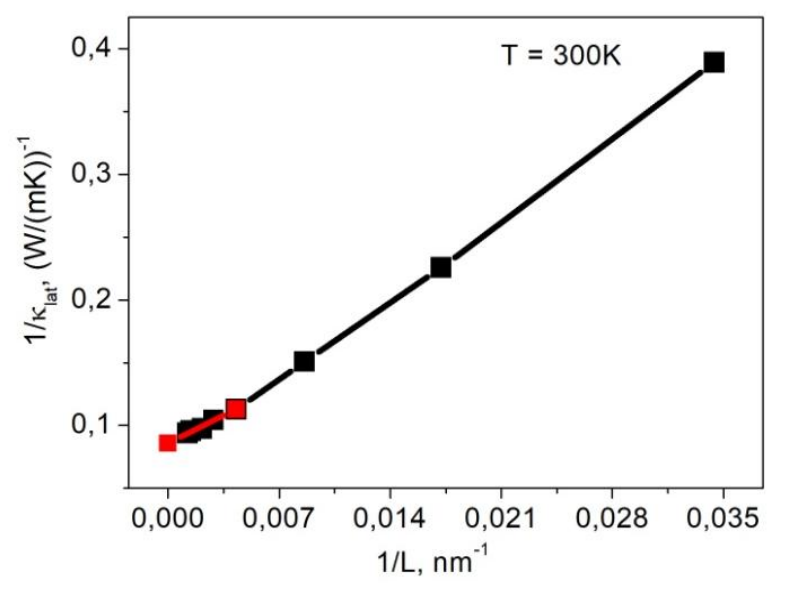

Fig. 4. The dependence of the reciprocal of the thermal conductivity on the reciprocal of the size of the region for the temperature $\mathrm{T}=300 \mathrm{~K}$.

To overcome the effects of the finite size, in accordance with the scaling procedure, the heat flux was determined by a series of calculations for various sizes of the computational 
domain. The number of computational regions and their sizes depended on the temperature for which the thermal conductivity was calculated. The lower the temperature, the more areas were selected. For the range $300 \mathrm{~K} \leq T \leq 900 \mathrm{~K}$, the calculations were carried out for 8 regions of different sizes $L_{n}: 20,40,80,160,240320,480,560$ unit cells corresponding to the number of particles. For the range $1200 \mathrm{~K} \leq T \leq 2000 \mathrm{~K}, 3$ calculations were performed for the sizes of the $L_{n}$ region: $80,160,240$ cells, and for $T \geq 4000 \mathrm{~K}$, only one calculation was performed for the size of the region $L_{n}=80$ cells (for larger size, the results were the same). The cross section of the region was constant: $S=10 \times 10$ cells. The heat flux was determined from the temperature difference between the heating and heat sink areas, for which the instantaneous temperature difference was averaged over the entire calculation time after establishing a stationary distribution. To increase the accuracy of the calculations, the temperature difference was calculated not over the entire interval between the source and the sink, but in its central part, 0.8 of the total length.After a series of calculations, for each temperature from the range $300 \mathrm{~K}<T<5700 \mathrm{~K}$, the scaling procedure was performed and thermal conductivity was calculated. Let us consider the scaling procedure using the example of calculating the thermal conductivity of copper for $300 \mathrm{~K}$. Figure 4 shows the dependence of the reciprocal of the thermal conductivity on the reciprocal of the size of the region for a temperature of $300 \mathrm{~K}$. The results of the calculations on the graph are shown by black lines with markers.

Five values of the inverse thermal conductivity corresponding to the longest lengths of the computational domain were approximated by a linear dependence (1)

$$
\frac{1}{\kappa_{\text {lat }}}(x)=0.086+6.13 x,
$$

where $x=1 / L_{n}$. The approximation error, according the least squares criterion (2) was $\Delta=$ 0.972. At $x=1 / L_{n}=0$ from the dependence (6) one can obtain the inverse value of thermal conductivity $1 / \kappa_{\text {lat }}=0.086$, from which the thermal conductivity of copper was obtained $\kappa_{\text {lat }}(T=300 \mathrm{~K})=11.627 \mathrm{~W} / \mathrm{mK}$. It corresponds to the infinite value of $L_{n}$. Fig.4 shows the linear dependence (6) as a red line with markers.

The results of calculation of the thermal conductivity of copper are presented in Fig. 5. The vertical dashed lines indicate the melting temperature $T_{m}$ and the critical temperature $T_{c r}$ of copper. At the temperature of $300 \mathrm{~K}$, according to the above calculations, the phonon thermal conductivity is $\kappa_{\text {lat }}=11.627 \mathrm{~W} / \mathrm{mK}$. With increasing temperature, the thermal conductivity of copper decreases. The region of the solid - liquid phase transition is shown in enlarged form in the inset of Fig. 5. It is seen that at the equilibrium melting temperature $T_{m}=1330 \mathrm{~K}$, the value of thermal conductivity decreases stepwise. In the solid phase, the thermal conductivity is $\kappa_{\text {lat }}=1.55 \mathrm{~W} / \mathrm{mK}$, and in the liquid phase at the same temperature the thermal conductivity is $\kappa_{\text {lat }}=1.29 \mathrm{~W} / \mathrm{mK}$. The difference in thermal conductivity between solid and liquid phases is $16.77 \%$. The calculation was carried out up to the temperature $T=5700 \mathrm{~K}$, at which the thermal conductivity is $\kappa_{\text {lat }}=0.647 \mathrm{~W} / \mathrm{mK}$. Such a change in the phonon thermal conductivity with increasing temperature does not contradict the ideas about the behavior of the phonon thermal conductivity of metals. In the near-critical region in Fig. 5. the dashed line shows the extrapolation of the temperature dependence of thermal conductivity to the critical point.

A comparison with alternative ab-inito calculations in the range $300 \mathrm{~K} \leq T<1000 \mathrm{~K}$ made by the Generalized Gradient Approximation (GGA) method in [36] showed good agreement. At 
low temperatures $(300 \mathrm{~K} \leq T<600 \mathrm{~K})$, the largest difference with [36] is $\Delta \kappa \sim 14 \%$ at $300 \mathrm{~K}$. With increasing temperature, the difference in results becomes smaller. At $T=600 \mathrm{~K}$, the difference is $\Delta \kappa \sim 4 \%$, and at $T=1000 \mathrm{~K}, \Delta \kappa \sim 2 \%$ - the results almost completely match. In the temperature range $T>1000 \mathrm{~K}$, there is no data for comparison. In general, such comparison results suggest that the selected method and potential describe the model with good accuracy and are applicable for further studies.

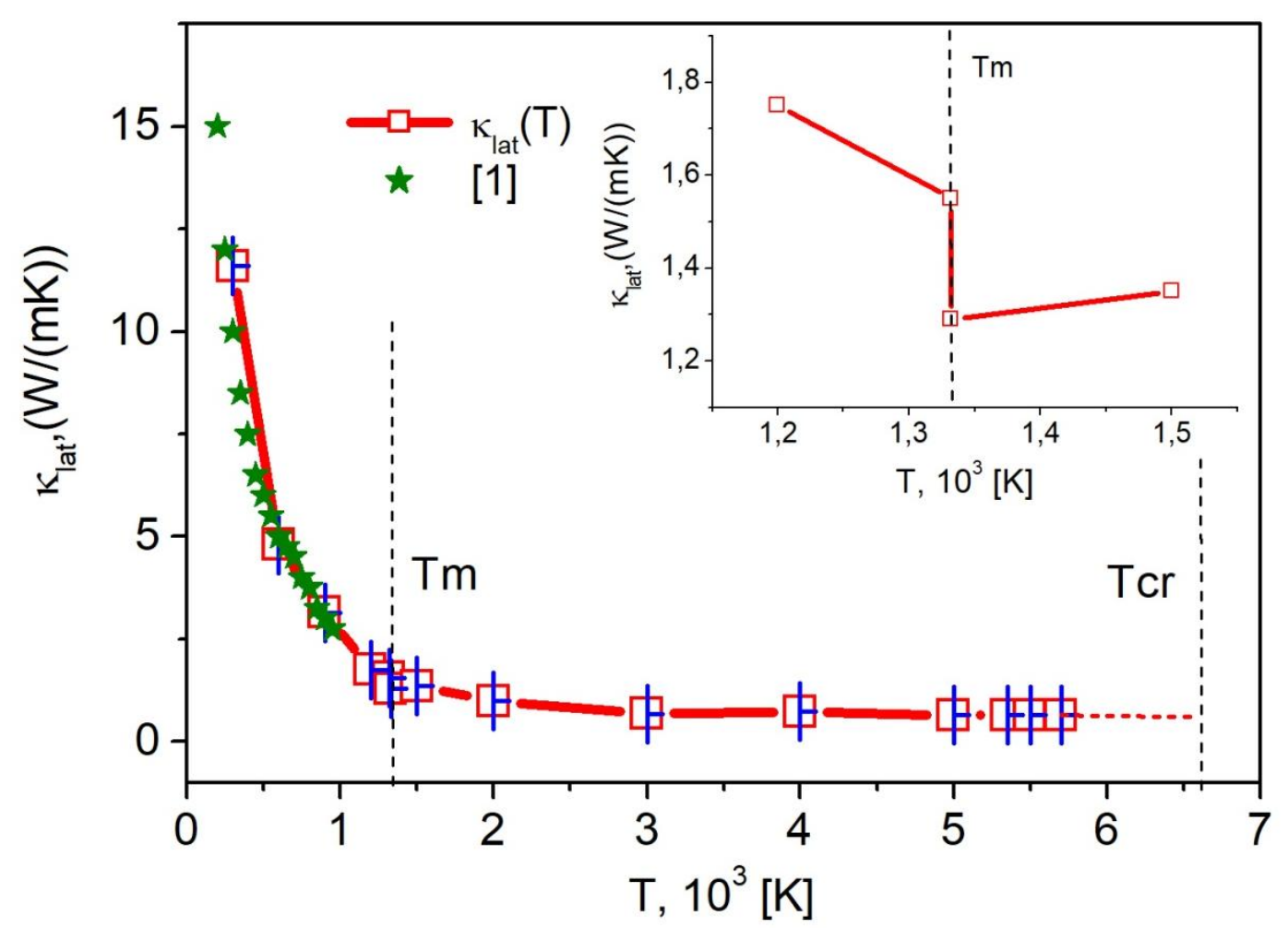

Fig. 5. Temperature dependence of the phonon thermal conductivity of copper. Markers [1] show the results of calculations from [36]. The fragment shows an abrupt decrease in thermal conductivity at the solid - liquid phase transition.

For the use in further calculations, the results obtained are more convenient to write as an analytical dependence of the form (1). For the solid phase, the results were approximated by a polynomial dependence of the 4 th degree, for the liquid phase by a polynomial of the $3 \mathrm{rd}$ degree

$$
\begin{gathered}
\kappa_{\text {lat }, \text { sol }}(T)=a_{0}+a_{1}\left(T-T_{0}\right)+a_{2}\left(T-T_{0}\right)^{2}+a_{3}\left(T-T_{0}\right)^{3}+a_{4}\left(T-T_{0}\right)^{4}, \\
\kappa_{\text {lat }, \text { liq }}(T)=a_{0}+a_{1}\left(T-T_{0}\right)+a_{2}\left(T-T_{0}\right)^{2}+a_{3}\left(T-T_{0}\right)^{3}
\end{gathered}
$$

where $T_{0}=300 \mathrm{~K}$ for the solid phase $\left(300 \mathrm{~K} \leq T \leq T_{m}\right), T_{0}=T_{m}$ for the liquid phase $\left(T_{m} \leq T \leq\right.$ $5700 K)$. The values of the coefficients $a_{k}$ and approximation errors according to the least squares criterion (2) are shown in the Table 3. 


\begin{tabular}{|l|l|l|}
\hline \multicolumn{1}{|c|}{$\mathrm{k}$} & solid & liquid \\
\hline$a_{0}$ & 11.627 & 1.29 \\
\hline$a_{1}$ & $-4.2781 \times 10^{-2}$ & $-5.01 \times 10^{-4}$ \\
\hline$a_{2}$ & $9.2403 \times 10^{-5}$ & $1.31 \times 10^{-7}$ \\
\hline$a_{3}$ & $-9.6577 \times 10^{-8}$ & $-1.2 \times 10^{-11}$ \\
\hline$a_{4}$ & $3.686 \times 10^{-11}$ & \\
\hline$\Delta\left(P\left(x_{j}\right), y_{j}\right)$ & $1.201 \times 10^{-11}$ & 0.021 \\
\hline
\end{tabular}

Table 3. The values of the coefficients $a_{k}$ of the function, which approximates the calculation results of the thermal conductivity of copper $\kappa_{\text {lat }}(T), W \cdot \mathrm{m}^{-1} \cdot \mathrm{K}^{-1}$

\section{CONCLUSION}

The temperature dependences were obtained for the thermophysical characteristics of copper: phonon thermal conductivity, heat capacity and density using molecular dynamics simulation with the EAM potential [21]. The wide temperature range $300 \mathrm{~K} \leq T \leq 5800 \mathrm{~K}$, in which the thermophysical properties of copper were determined in this work, covers the firstorder phase transition (melting-crystallization) and the near-critical region in which drastic changes in the thermophysical properties of the substance occur. The calculation results were approximated by polynomials of low degrees.

The temperature dependences of the density $\rho(T)$ and specific heat $C_{p}(T)$ of copper were determined from a series of molecular dynamics calculations within the framework of one computational experiment.

The temperature dependence of the density of copper $\rho(T)$ was obtained in the range $300 \mathrm{~K}<T<5620 \mathrm{~K}$. At the melting point, the density is calculated for two states of matter: solid and liquid. Based on the simulation results, the temperature range of the overheating of the solid phase $T_{m}<T<1.2 T_{m}$ and the density values in this range were obtained. The results obtained show good agreement with the experimental results [42].

The temperature dependence of the specific heat of copper $C_{p}(T)$ was obtained in the range $300 \mathrm{~K}<T<5800 \mathrm{~K}$. In the region of the solid - liquid phase transition at an equilibrium melting temperature $T_{m}$, a small stepwise decrease in the heat capacity of copper occurs, amounting to $\sim 3.128 \%$ (according to the experiment [38], 1.529\%). In the temperature range of the overheating of the solid phase $T_{m}<T<1.2 T_{m}$, the specific heat was obtained. In the temperature range $T_{m}<T<2.63 T_{m}$ in the liquid phase, the specific heat is constant and amounts to $C_{p}(T) \approx$ $31.0 \mathrm{~J} \cdot \mathrm{mol}^{-1} \cdot \mathrm{K}^{-1}$, which is $8 \%$ less than in [38]. At temperatures above $4000 \mathrm{~K}$, the heat capacity of copper increases and at $T=5800 \mathrm{~K}$ its value is $C_{p}(T)=47.698 \mathrm{~J} \cdot \mathrm{mol}^{-1} \cdot \mathrm{K}^{-1}$. The results obtained show good agreement with the experimental results $[37,38]$.

Based on a series of calculations by the direct method using molecular dynamics simulation, the temperature dependence of the phonon thermal conductivity of copper was obtained in the temperature range $300<T<5700 \mathrm{~K}$. The EAM potential [21] was used in the simulation. In the region of the solid - liquid phase transition, at the equilibrium melting temperature $T_{m}=1330 \mathrm{~K}$, the value of thermal conductivity decreases stepwise. The difference in thermal conductivity between solid and liquid phases is $16.77 \%$. Comparison with alternative ab-inito calculations in the range $300 \mathrm{~K} \leq T<1000 \mathrm{~K}$ made by the Generalized Gradient Approximation (GGA) method in [36] showed good agreement. 
In general, such comparison results suggest that the selected interparticle interaction potential and calculation methods for copper of the phonon specific heat, phonon thermal conductivity, and density show good accuracy and can be used for further studies.

Acknowledgements: This study was supported by the Russian Science Foundation (project no. 18-11-00318).

\section{REFERENCES}

[1] Mónica Fernández-Arias, Mohamed Boutinguiza, Jesús del Val, Antonio Riveiro, Daniel Rodríguez, Felipe Arias-González, Javier Gil and Juan Pou, "Fabrication and Deposition of Copper and Copper Oxide Nanoparticles by Laser Ablation in Open Air", Nanomaterials, 10, 300 (2020).

[2] S. Fernando, T. Gunasekara, J. Holto, "Antimicrobial Nanoparticles: applications and mechanisms of action", Sri Lankan Journal of Infectious Diseases, 8(1), 2-11 (2018)

[3] Ali K. Yetisen, Yunuen Montelongo, Fernando da Cruz Vasconcellos, J.L. Martinez-Hurtado, Sankalpa Neupane, Haaider Butt, Malik M. Qasim, Jeffrey Blyth, Keith Burling, J. Bryan Carmody, Mark Evans, Timothy D. Wilkinson, Lauro T. Kubota, Michael J. Monteiro, and Christopher R. Lowe, "Reusable, Robust, and Accurate Laser-Generated Photonic Nanosensor", Nano Lett., 14, 3587-3593 (2014).

[4] Muhammad Imran Din, Rida Rehan, "Synthesis, characterization, and applications of copper nanoparticles", Analytical Letters, 50(1), 50-62, (2017).

[5] Amir Reza Sadrolhosseini, Mohd Adzir Mahdi, Farideh Alizadeh and Suraya Abdul Rashid, "Laser Ablation Technique for Synthesis of Metal Nanoparticle in Liquid", Chapter 4 in Laser Technology and its Applications, Yufei Ma (Ed.), InTech (2020).

[6] S. Barcikowski, A. Hahn, A. V. Kabashin, and B. N. Chichkov, "Properties of nanoparticles generated during femtosecond laser machining in air and water," Appl. Phys. A, 87, 47-55 (2007).

[7] P.V. Kazakevich, V.V. Voronov, A.V. Simakin, G.A. Shafeev. "Production of copper and brass nanoparticles upon laser ablation in liquids", Quantum Electronics, 34(10), 951-956 (2004)

[8] N. Tsakiris, K. K. Anoop, G. Ausanio, M. Gill-Comeau, R. Bruzzese, and S. Amoruso, "Ultrashort laser ablation of bulk copper targets: Dynamics and size distribution of the generated nanoparticles," J. Appl. Phys., 115, 243301 (2014)

[9] C. M. Rouleau, C.-Y. Shih, C. Wu, L. V. Zhigilei, A. A. Puretzky, and D. B. Geohegan, "Nanoparticle generation and transport resulting from femtosecond laser ablation of ultrathin metal films: Time-resolved measurements and molecular dynamics simulations," Appl. Phys. Lett., 104, 193106 (2014).

[10] A. Amouye Foumani, A. R. Niknam, "Atomistic simulation of femtosecond laser pulse interactions with a copper film: Effect of dependency of penetration depth and reflectivity on electron temperature", J. of Appl. Phys., 123, 043106 (2018)

[11] V.I. Mazhukin, A.V. Shapranov, A.V. Mazhukin, O.N. Koroleva, "Mathematical formulation of a kinetic version of Stefan problem for heterogeneous melting/crystallization of metals", Mathematica Montisnigri, 36, 58-77 (2016)

[12] A.A. Samokhin, V.I. Mazhukin, A.V. Shapranov, M.M. Demin, A.E. Zubko, "Molecular dynamics modeling of nanosecond laser ablation: transcritical regime", Mathematica Montisnigri, 38, 78-89 (2017) 
[13] V. I. Mazhukin, A. V. Mazhukin, M. M. Demin, A. V. Shapranov. "Nanosecond laser ablation of target $\mathrm{Al}$ in a gaseous medium: explosive boiling". Applied Physics A: Material Science and Processing, 124 (3), 237(10), (2018)

[14] V.I. Mazhukin, M.M. Demin, A.V. Shapranov. "High-speed laser ablation of metal with pico- and subpicosecond pulses". Applied Surface Science, 302, 6-10 (2014).

[15] C. Wu and L. V. Zhigilei, "Microscopic mechanisms of laser spallation and ablation of metal targets from large-scale molecular dynamics simulations", Appl. Phys. A, 114, 11-32 (2014).

[16] Neil W. Ashcroft, N. David Mermin, Solid State Physics, Holt, Rinehart and Winston, (1976).

[17] Z. Tong, S. Li, X. Ruan, and H. Bao, "Comprehensive first-principles analysis of phonon thermal conductivity and electron-phonon coupling in different metals", Physical review, B 100, 144306 (2019)

[18] A.V. Mazhukin, V.I. Mazhukin, M.M. Demin, "Modeling of femtosecond laser ablation of Al film by laser pulses", Applied Surface Science, 257, 5443-5446 (2011).

[19] R. Venkatasubramanian, E. Siivola, T. Colpitts and B. O. Quinn, "Thin Film Thermoelectric Devices with High Room Temperature Figures of Merit", Nature, 413, 597602 (2001). http://dx.doi.org/10.1038/35098012

[20] M.M. Demin, V.I. Mazhukin, A.A. Aleksashkina, "Molecular dynamic calculation of lattice thermal conductivity of gold in the melting-crystallization region", Mathematical Montisnigri, 46, 105-113 (2019)

[21] Y. Mishin, M. J. Mehl and D. A. Papaconstantopoulos, A. F. Voter, J. D. Kress, "Structural stability and lattice defects in copper: Ab initio, tight-binding, and embedded-atom calculations", Phys. Rev. B, 63, 224106 (2001)

[22] S.M. Foiles, M.I. Baskes, and M.S. Daw, "Embedded-atom-method functions for the fcc metals Cu, Ag, Au, Ni, Pd, Pt, and their alloys", Physical Review B, 33(12), 7983-7991 (1986). DOI: $10.1103 /$ physrevb.33.7983.

[23] S.V. Starikov, A.Y. Faenov, T.A. Pikuz, I.Y. Skobelev, V.E. Fortov, S. Tamotsu, M. Ishino, M. Tanaka, N. Hasegawa, M. Nishikino, T. Kaihori, T. Imazono, M. Kando, and T. Kawachi, "Soft picosecond X-ray laser nanomodification of gold and aluminum surfaces", Applied Physics B, 116(4), 1005-1016 (2014). DOI: 10.1007/s00340-014-5789-y.

[24] P. K. Schelling, S. R. Phillpot, P. Keblinski, "Comparison of atomic-level simulation methods for computing thermal conductivity", Phys. Rev. B, 65, 144306 (2002)

[25] L. Hu, W. J. Evans, P. Keblinski, "One-dimensional phonon effects in direct molecular dynamics method for thermal conductivity determination", J. Appl. Phys., 110, $113511(2011)$

[26] Florian Müller-Plathe, "A simple nonequilibrium molecular dynamics method for calculating the thermal conductivity", J. Chem. Phys., 106, 6082 (1997).

[27] V.I. Mazhukin, M.M. Demin, A.A. Aleksashkina, "Atomistic modeling of thermophysical properties of cooper in the region of the melting point", Mathematical Montisnigri, 41, 99-111 (2018)

[28] V.S. Dozhdikov, A.Y. Basharin, P.R. Levashov, "Two-phase simulation of the crystalline silicon melting line at pressures from -1 to 3 GPa”, J. Chem. Phys., 137(5), 054502 (2012)

[29] Yang Chun Zou, Shi Kai Xiang, Cheng Da Dai. "Investigation on the efficiency and accuracy of methods for calculating melting temperature by molecular dynamics simulation", Computational Materials Science, 171, 109156(1-11) (2020).

[30] M.M. Demin, O.N. Koroleva, A.V. Shapranov, A.A. Aleksashkina, "Atomistic modeling of the critical region of copper using a liquid-vapor coexistence curve", Mathematical Montisnigri, 46, 61-71 (2019) 
[31] O.N. Koroleva, M.M. Demin, V.I. Mazhukin, A.V. Mazhukin, "Modeling of thermal conductivity of $\mathrm{Si}$ in the range from the normal to near-critical conditions", Mathematical Montisnigri, 45, 85-94 (2019)

[32] G. Sebastian, Gang Chen Volz, "Molecular-dynamics simulation of thermal conductivity of silicon crystals", PHYS. REV. B, 61(4), 2651-2656 (2010)

[33] D.P. Sellan, E.S. Landry, J.E. Turney, A.J.H. McGaughey, and C.H. Amon, "Size effects in molecular dynamics thermal conductivity predictions", PHYS. REV. B, 81, 214305 (2010)

[34] P.C. Howell, "Comparison of molecular dynamics methods and interatomic potentials for calculating the thermal conductivity of silicon", J. Chem. Phys., 137, 224111 (2012).

[35] L. Verlet, "Computer "Experiments" on Classical Fluids. I. Thermodynamically Properties of Lennard-Jones Molecules", Phys. Rev., 159, 98-103 (1967).

[36] Y. Wang, Z. Lu, and X. Ruan, "First principles calculation of lattice thermal conductivity of metals considering phonon-phonon and phonon-electron scattering", Journal of applied physics, 119, 225109 (2016)

[37] Fizicheskie velichiny, Spravochnik pod red. I.S. Grigoreva, E.Z. Meilihova, M: Energoatomizdat (1991)

[38] M. W. Chase, Jr., C. A. Davies, J. R. Downey, Jr., D. J. Frurip, R. A. McDonald, and A. N. Syverud, "JANAF Thermochemical Tables, Third Edition", J. Phys. Chem. Ref. Data, 14(1), (1985)

[39] S. Plimpton, "Fast parallel algorithms for short-range molecular dynamics", $J$. Comput. Phys., 117(1), 1-19 (1995)

[40] H.J.C. Berendsen, J.P.M. Postma, W.F. Van Gunsteren, A. DiNola, J.R. Haak, "Molecular dynamics with coupling to an external bath", J. Chem. Phys., 81, 3684-3690 (1984).

[41] A.A. Samarskii, F.I. Gulin, Chislennye metody, M.: Fizmatlit, (1989)

[42] J.A. Cahill, A. D. Kirshenbaum, "The density of liquid copper from its melting point (1356K) to $2500 \mathrm{~K}$ and an estimate of its critical constants", J. Phys. Chem., 66, 1080-1082 (1962)

[43] P.M. Nasch and S.G. Steinemann, "Density and thermal expansion of molten manganese, iron, nickel, copper, aluminum and tin by means of the gamma-ray attenuation technique", Phys. Chem. Liq., 29, 43 (1995).

[44] M.J. Assael, A.E. Kalyva, K.D. Antoniadis, R.M. Banish, I. Egry, J. Wu, E. Kaschnitz, W.A. Wakeham, "Reference Data for the Density and Viscosity of Liquid Copper and Liquid Tin", J. of Phys. and Chem., 39, 033105 (2010)

[45] G.T. Furukawa, W.G. Saba, M.L. Reilly, Critical analysis of the heat-capacity data of the literature and evaluation of thermodynamic properties of copper, silver, and gold from 0 to $300 \mathrm{~K}$, National Standard Reference Data SeriesNational Bureau of Standards 18, Washington, p. 64 (1968).

[46] R. Hultgren, P.D. Desai, D.T. Hawkins, M. Gleiser, K. K. Kelley, and D. D. Wagman, Selected Values of the Thermodynamic Properties of the Elements, Am. Soc. Metals, Metals Park, OH, (1973).

[47] A.K. Chaudhuri, D.W. Bonnell, L.A. Ford, J.L. Margrave, High Temp. Sci, 2(3), 203$212(1970)$

Received November 15, 2019 